\title{
Patterns of heart rate variability and cardiac autonomic modulations in controlled and uncontrolled asthmatic patients
}

\author{
Mohamed Faisal Lutfi
}

\begin{abstract}
Background: Previous heart rate variability (HRV) studies in asthmatic subjects (AS) demonstrate predominance of parasympathetic drive concomitant with low HRV, which is against the general belief that enhanced parasympathetic modulation improves HRV. The aim of this study was to compare patterns of HRV and cardiac autonomic modulations of AS to healthy control subjects (HS).
\end{abstract}

Methods: Eighty AS and forty HS were enrolled in the study. Asthma control test and spirometry were used to discriminate uncontrolled (UA) from controlled (CA) asthmatic patients. Natural logarithmic (Ln) scale of total power (TP), very low frequency (VLF), low frequency (LF) and high frequency (HF) were used to evaluate HRV. Normalized low frequency (LF Norm) and high frequency (HF Norm) were used to determine sympathetic and parasympathetic autonomic modulations respectively.

Results: CA patients achieved significantly higher LnTP, LnLF, LnHF and HF Norm but lower LF Norm and LnLF/HF compared with UA patients $(p<0.05)$. Although CA patients showed increased HRV and augmented vagal modulation compared with $\mathrm{HS}$, these findings were no longer significant following adjustment for mean heart rates and anti-asthma treatment. All measured HRV parameters were not significantly different in UA patients compared with the HS $(p>0.05)$.

Conclusions: CA is associated with enhanced parasympathetic modulations and higher HRV compared with UA. However, neither CA nor UA patients had different autonomic modulations and/or HRV compared with HS.

Keywords: Asthma control, Autonomic modulation, Heart rate variability

\section{Background}

Assessment of heart rate variability (HRV) is one of the most popular methods used to evaluate autonomic modulations of the heart [1]. Recent research exploring deranged autonomic functions in certain diseases was largely based on HRV studies [2-4]. Bronchial asthma has received special attention by researchers investigating the impact of autonomic disturbances on the disease etiology [5-11]. Low HRV and predominance of parasympathetic modulations on the heart were demonstrated in some studies [5-8], but not others [9-11]. According to some previous reports, parasympathetic discharge seems to predominate in asthmatic patients irrespective of day-and-night cycle [12] or physical activity

Correspondence: mohamedfaisallutfi@gmail.com

Department of Physiology, Faculty of Medicine and Health Sciences, Al-Neelain University, Khartoum, Sudan
[5]. Conversely, some studies were unable to prove vagal hypertonia in patients with bronchial asthma [6]. Based on HRV derived indices, Gupta et al. failed to demonstrate parasympathetic predominance in asthmatic patients; however, their results suggested sympathetic withdrawal among the studied patients [6]. Garcia-Araújo and colleagues compared the autonomic modulation in 14 controlled asthmatic patients and 10 healthy volunteers at rest and during exercise. Their results suggested that controlled asthmatic patients had an increased sympathetic modulation and attenuated response to the postural changes [9]. In another study, HRV and cardiac autonomic modulations in difficult-to-control asthma patients were not different compared with healthy subjects [10].

In clinical practice, low HRV suggests increased susceptibility to cardiac arrhythmias secondary to autonomic imbalance [13, 14]. Enhanced parasympathetic 
drive is expected to increase HRV. Nonetheless, most studies in asthmatic patients that document shift of sympathovagal balance towards predominance of parasympathetic drive also demonstrate reduced HRV [5-8]. These contradictory findings are difficult to explain physiologically and may point to hidden confounders [15-18]. From a physiological standpoint, exploring variations in HRV in asthmatic patients should consider differences in heart rate $[15,16]$ and medical treatments $[18,19]$ among these patients. Altered heart rate is expected in asthmatic patients as a result of activation of the chemoreceptor reflex triggered by hypoxia/hypercapnia; sympathetic stimulation triggered by the stress of asthma attack; and beta- 2 agonist therapy. Alternatively, heart rate is inversely proportional to beat-to-beat intervals, from which all HRV parameters are derived. Consequently, results of studies that do not adjust for asthma-associated changes in heart rates, and anti-asthma treatments (AAT), while comparing HRV and autonomic modulations between asthmatic and non-asthmatic subjects are questionable and deserve re-evaluation. Previous reports that failed to show predominance of vagal drive in asthmatic individuals may be biased by heterogeneous patterns of autonomic modulations among asthmatic patients [9-11]. Poor response of difficult-to-control asthma patients to anticholinergic drugs raises the question of whether enhanced vagal drive contributes to the pathogenesis of asthma in these patients $[19,20]$. A negative answer is suggested by a recent study, which fails to demonstrate significant difference in the autonomic modulations between difficultto-control asthma patients and healthy control subjects [10]. Further studies are required to verify if parasympathetic drive predominates in all asthmatic patients regardless of their disease control, AAT and asthma-associated changes in heart rates. The aim of this study was to compare patterns of HRV and cardiac autonomic modulations of asthmatic patients to healthy control subjects, taking into consideration possible influences of degree of asthma control, AAT and asthma-associated changes in heart rates on HRV.

\section{Methods}

The study received ethical clearance from the ethics review committee at the Faculty of Medicine, University of Khartoum, Sudan. All volunteers provided informed written consent before being involved in the study.

Eighty (41 males and 39 females) known asthmatic subjects (AS) with no past medical history of other chronic pulmonary diseases were matched for age, gender, weight and height with a control group of 40 (22 males and 18 females) apparently healthy subjects (HS). All studied individuals were young adults, and had no past medical history of smoking, diabetes mellitus, hypertension, heart disease or other diseases that may affect cardiac autonomic modulations. The test group was recruited from respiratory referral clinics of the Khartoum teaching hospitals, Sudan. The control subjects were students and doctors of the Faculty of Medicine and Health Sciences, Al-Neelain University, Khartoum, Sudan.

Following evaluation of past medical history, including intensity of anti-asthma treatment offered to the patients, all volunteers underwent clinical examination and investigations during the period from $09.00 \mathrm{AM}-12.00$ PM to avoid confounding effects of circadian rhythm on asthma severity, spirometry and HRV. Using asthma control test (ACT), a score of 15 or less was used to discriminate uncontrolled (UA) from controlled asthmatic (CA) patients [21, 22]. For further verification of ACT, ventilatory function was evaluated in each class using spirometry. For the purpose of adjustment for anti-asthma medications, patients were further categorized into: offtreatment patients, patients on beta- 2 agonists only and patients on combined beta- 2 agonists and steroids. A GIMA scale (GIMA S.p.A, Milan, Italy) was used to measure weight and height. Body mass index (BMI) was determined by the formula: BMI $\left(\mathrm{kg} / \mathrm{m}^{2}\right)=$ weight $/$ height squared. Spirometry and HRV were performed according to the standard methods using Allflow Spirometer (Version 5.18, Clement Clarke International Limited, Harlow, UK) and Biocom 3000 ECG recorder (Heart Rhythm Scanner, Version 2.0, Biocom Technologies, Poulsbo, WA, U.S.A) respectively [15, 23].

HRV parameters were derived from 5-min electrocardiogram (ECG) recordings in the supine position after ensuring clean ECG signals, absence of movement artifacts and comfortable breathing. Frequency domain analysis was used to determine HRV and cardiac autonomic modulations in the different groups. Natural logarithm (Ln) of total power (LnTP), very low frequency (LnVLF), low frequency (LnLF) and high frequency (LnHF) were used to evaluate HRV. Alternatively normalized low frequency (LF Norm) and high frequency (HF Norm) were used to determine sympathetic and parasympathetic autonomic modulations respectively [24]. The Biocom 3000 ECG recorder also calculated the mean heart rate (MHR) at the time of ECG recording.

\section{Statistical analysis}

Statistical analysis was done using SPSS for windows (Version 16; Chicago, IL, USA). Variables were described by means, median, standard deviations (SD), 25 quartile (Q1) and 75 quartile (Q3) based on their distribution curves. Using appropriate statistical tests, comparable distributions of gender, ages and BMI among control and test groups were established. Concordance of spirometric measurements with ACT groups was ensured to approve proper grading of asthma severity by ACT. Means (SD) and median (Q1-Q3) of frequency domain 
HRV parameters and spirometric measurements were compared in the studied groups using one-way ANOVA (Student's $T$ test for comparison between two groups) and Kruskal-Wallis (Mann-Whitney $U$ test for comparison between two groups) tests, respectively. Using a general linear model MHR, and AAT for each subject were introduced as covariates while comparing each HRV parameter in the different studied groups. A value of $P<0.05$ was considered significant.

\section{Results}

Of the AS $(n=80), 37[46.3 ; 95 \%$ confidence interval (CI): 35.8, $57.1 \%$ ] had CA and 43 (53.7; $95 \%$ CI: 42.9, $64.3 \%)$ had UA. The distribution of gender $\left(c h i^{2}=0.15\right.$, $p=0.698)$, ages, and BMI were comparable in AS and HS. In contrast, MHR was significantly lower in HS [83.6 (11.3) beats/min] compared with CA [90.3 (10.6) beats $/ \mathrm{min}, p=0.012]$ and UA [88.6 (12.3) beats $/ \mathrm{min}, p=$ 0.048] (Fig. 1). As shown in Table 1, all measured spirometric indices were significantly increased in HS compared with CA and UA. Except for peak expiratory flow rate, the same spirometric measurements were significantly increased in CA compared with UA.

CA patients achieved significantly higher LnTP, LnLF, LnHF and HF Norm [7.40 (1.27) $\mathrm{ms}^{2} / \mathrm{Hz}, 6.23$ (1.30) $\mathrm{ms}^{2} / \mathrm{Hz}, 6.41(1.65) \mathrm{ms}^{2} / \mathrm{Hz}$ and 54.4 (16.4) normalized unit (nu) respectively] compared with the HS [6.68 (1.31) ms, $p=0.023 ; 5.50$ (1.43), $p=0.026 ; 5.32$ (1.76) ms, $p=0.008$ and 45.9 (17.3) nu, $p=0.026$ respectively] as well as UA patients [median 6.64 (1.49) ms, $p=0.015$; 5.53 (1.47) ms, $p=0.030 ; 5.31$ (1.84) ms, $p=0.006$ and 45.8 (17.6) nu, $p=0.026$ respectively] (Table 2 ).

LF Norm and LnLF/HF were significantly lower in CA patients [45.6 (16.4) nu and -0.19 (0.75) respectively] compared with the HS [54.1 (17.3) nu, $p=0.021$ and
$0.19(0.76), p=0.040$ respectively] as well as UA patients [54.2 (17.6) nu, $p=0.041$ and 0.22 (0.86), $p=0.022$ respectively]. All measured frequency domain HRV parameters were not significantly different in UA patients compared with HS (Table 2).

All HRV differences between the groups were still significant following adjustment for MHR at the time of HRV recording, except for LF Norm, HF Norm and LnLF/HF, which became comparable in HS and CA patients (Table 2). Adjustment for MHR and AAT nullified all significant variations between $\mathrm{HS}$ and $\mathrm{CA}$ but all other differences between CA and UA patients remained statistically significant (Table 2).

\section{Discussion}

It is evident from the present results that global HRV and autonomic modulations of CA patients are significantly different from UA patients irrespective of the possible influences of MHR and AAT on HRV. Based on absolute and normalized HRV parameters, CA patients had higher global HRV and augmented parasympathetic autonomic modulations compared with UA patients. Comparing HS to AS, it is clear that global HRV and autonomic modulations of UA patients were comparable to the HS group regardless of influences of MHR and AAT on HRV. Although CA patients showed higher HRV and augmented vagal modulation compared with HS, these findings were no longer significant following adjustment for MHR and AAT.

Several previous reports confirm predominance of the parasympathetic nervous system on cardiac autonomic modulations of asthmatic patients [5, 6, 25-27]. Gupta et al. compared findings of spectral analysis HRV of 30 asthmatic patients aged 20-30 years to a similar number of healthy volunteers. Their results confirmed lower LF
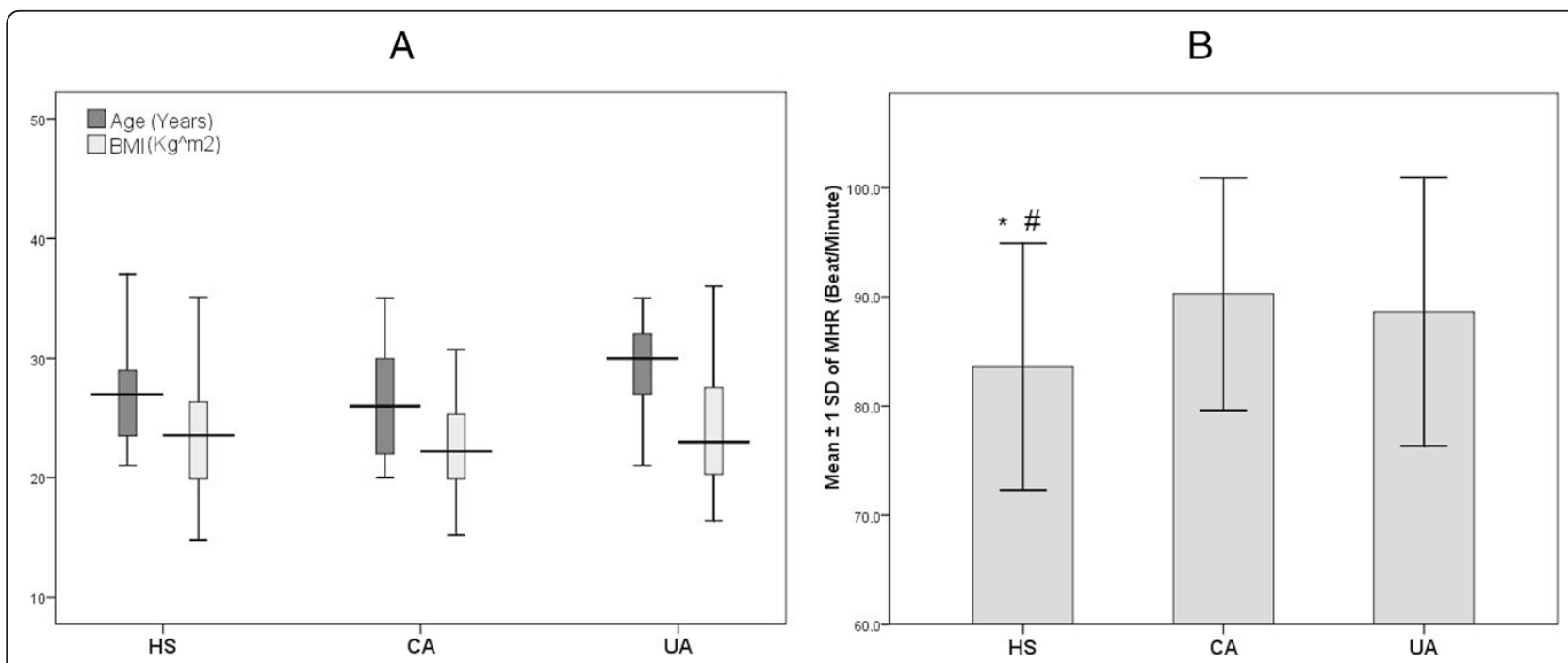

Fig. 1 Distribution of age, BMI (a) and MHR (b) among studied groups. * Significant compared with CA, \# significant compared with UA 
Table 1 ACT score and spirometric measurements among studied groups

\begin{tabular}{|c|c|c|c|c|}
\hline & $\mathrm{HS}$ & CA & UA & $P$ \\
\hline & $N=40$ & $N=37$ & $N=43$ & \\
\hline & Mean (SD) & Mean (SD) & Mean (SD) & \\
\hline & Median (Q1 -Q3) & Median (Q1 -Q3) & Median (Q1 -Q3) & \\
\hline ACT score & & $19.0(17.0-21.0)$ & $13.0(10.0-13.0)$ & $<0.001^{*}$ \\
\hline \multirow[t]{3}{*}{ FEV1\% } & $90.5(5.5)$ & 78.5 (14.6) & $68.0(13.6)$ & HS vs. $C A<0.001^{*}$ \\
\hline & & & & HS vs. UA $<0.001^{*}$ \\
\hline & & & & CA vs. $U A<0.001^{*}$ \\
\hline \multirow[t]{3}{*}{ PEFR (L/sec) } & $7.4(1.6)$ & $5.3(1.9)$ & $4.5(1.9)$ & HS vs. $C A<0.001^{*}$ \\
\hline & & & & HS vs. UA $<0.001^{*}$ \\
\hline & & & & CA vs. $U A=0.054$ \\
\hline \multirow[t]{3}{*}{ FEF25\% (L/sec) } & $6.8(1.5)$ & $4.7(2.0)$ & $3.5(1.8)$ & HS vs. $C A<0.001^{*}$ \\
\hline & & & & HS vs. UA $<0.001^{*}$ \\
\hline & & & & CA vs. $U A=0.006^{*}$ \\
\hline \multirow[t]{3}{*}{ FEF50\% (L/sec) } & $4.9(3.7-6.1)$ & $2.8(2.0-4.4 .0)$ & $1.7(1.0-2.6)$ & HS vs. $C A<0.001^{*}$ \\
\hline & & & & HS vs. $U A<0.001^{*}$ \\
\hline & & & & CA vs. $U A=0.004^{*}$ \\
\hline \multirow[t]{3}{*}{ FEF75\% (L/sec) } & $2.3(1.8-2.5)$ & $1.2(0.7-1.8)$ & $0.7(0.4-1.3)$ & HS vs. $C A<0.001^{*}$ \\
\hline & & & & HS vs. UA $<0.001^{*}$ \\
\hline & & & & CA vs. $U A=0.005^{*}$ \\
\hline
\end{tabular}

ACT Asthma control test, CA Controlled asthmatic, FEF Forced expiratory flow, FEV1 Forced expiratory volume in the first second, HS Healthy subjects, PEFR Peak expiratory flow rate, Q1 25 quartile, Q3 75 quartile, SD Standard deviation, UA Uncontrolled asthma, * Statistically significant.

Norm in asthmatic patients compared with the healthy volunteers but failed to demonstrate significant difference in HF Norm among the studied groups [6]. These findings were further supported by the work of Gomes et al. who failed to demonstrate significant decrease in HF power following shuttle walk tests offered to asthmatic children. From an autonomic standpoint, the Gomes et al. findings suggested predominance of parasympathetic cardiac modulations even during exercise, when sympathetic effects were expected to dominate [5]. In another study, circadian rhythm of parasympathetic nervous function disappeared in 9 out of the 80 asthmatic children examined by Kazuma et al. but was observed in all healthy children [12]. Absence of parasympathetic circadian rhythm in some asthmatic children is probably due to persistent high parasympathetic discharge. Interestingly, enhanced parasympathetic cardiac modulations were proven in patients who are at higher risk to develop asthma, e.g., those with allergic rhinitis [28] and atopic dermatitis [29].

It is worth mentioning that the results of this study stand midway between the findings of previous researchers exploring autonomic modulations in asthmatic patients. This is because the present results suggested augmented parasympathetic and depressed sympathetic autonomic modulations in CA compared with UA patients but fail to demonstrate similar differences in autonomic trends when the AS group was compared with HS. To our knowledge, the present study is the first report to state clearly different patterns of cardiac autonomic modulations among controlled and uncontrolled asthmatic patients, although some previous reports gave cues that may support the present hypothesis $[10,11]$. Cabiddu et al. studied HRV in eight patients suffering from difficult-to-control asthma during different stages of sleep [10]. In healthy subjects in this study, HRV showed predominant parasympathetic drive during nonrapid eye movement sleep and an increased sympathetic activity during rapid eye movement sleep. Spectral analysis of HRV in difficult-to-control asthma patients showed trends in the main spectral indices comparable to the control subjects [10].

According to the present results, CA patients have higher global HRV compared with UA subjects but the HRV of either asthma group was comparable to HS. Conversely, previous reports repeatedly demonstrated reduced HRV among asthmatic patients compared with healthy individuals $[7,9,12,30]$. The incongruity of our results with previous reports might be explained by the unique design of our study which considered adjustment for MHR and AAT while comparing AS to HS. Alternatively, higher global HRV of CA compared with UA subjects could be explained by the findings of a recent study designed by Domnik et al. to assess the impact of 
Table 2 Comparison of HRV parameters between groups

\begin{tabular}{|c|c|c|c|c|c|c|}
\hline & $\mathrm{HS}$ & CA & UA & $P$ values & & \\
\hline & $N=40$ & $N=37$ & $N=43$ & Non-Adjusted & Adjusted for MHR & Adjusted for MHR and AAT \\
\hline & Mean (SD) & Mean (SD) & Mean (SD) & & & \\
\hline $\operatorname{LnTP}(\mathrm{ms} 2 / \mathrm{Hz})$ & $6.68(1.31)$ & $7.40(1.27)$ & $6.64(1.49)$ & HS vs. $C A=0.023^{*}$ & HS vs. $C A=0.037^{*}$ & HS vs. $C A=0.673$ \\
\hline & & & & HS vs. UA = 0.885 & HS vs. UA = 0.857 & HS vs. $\cup A=0.314$ \\
\hline & & & & CA vs. $U A=0.015^{*}$ & CA vs. $U A=0.019^{*}$ & CA vs. $U A=0.020^{*}$ \\
\hline $\operatorname{LnVLF}(\mathrm{ms} 2 / \mathrm{Hz})$ & $5.47(1.12)$ & $5.87(1.02)$ & $5.36(1.47)$ & HS vs. $C A=0.157$ & HS vs. $C A=0.163$ & HS vs. $C A=0.821$ \\
\hline & & & & HS vs. UA $=0.673$ & HS vs. UA $=0.950$ & HS vs. $U A=0.481$ \\
\hline & & & & CA vs.UA $=0.065$ & CA vs. $U A=0.076$ & CA vs. $U A=0.080$ \\
\hline $\operatorname{LnLF}(m s 2 / H z)$ & $5.50(1.43)$ & $6.23(1.30)$ & $5.53(1.47)$ & HS vs. $C A=0.026^{*}$ & HS vs. $C A=0.042^{*}$ & HS vs. $C A=0.658$ \\
\hline & & & & HS vs. $U A=0.911$ & HS vs. UA $=0.668$ & HS vs. UA $=0.141$ \\
\hline & & & & CA vs. $U A=0.030^{*}$ & CA vs. $U A=0.033$ & CA vs. $\cup A=0.031^{*}$ \\
\hline LnHF (ms2/Hz) & $5.32(1.76)$ & $6.41(1.65)$ & $5.31(1.84)$ & HS vs. $C A=0.008^{*}$ & HS vs. $C A=0.016^{*}$ & HS vs. $C A=0.490$ \\
\hline & & & & HS vs. UA = 0.979 & HS vs. UA = 0.761 & HS vs. $\cup A=0.346$ \\
\hline & & & & CA vs. $U A=0.006^{*}$ & CA vs. $U A=0.007^{*}$ & CA vs. $\cup A=0.008^{*}$ \\
\hline LF Norm (nu) & $54.1(17.3)$ & $45.6(16.4)$ & $54.2(17.6)$ & HS vs. $C A=0.021^{*}$ & HS vs. $C A=0.053$ & HS vs. $C A=0.406$ \\
\hline & & & & HS vs. UA $=0.748$ & HS vs. $U A=0.896$ & HS vs. UA $=0.716$ \\
\hline & & & & CA vs.UA $=0.041^{*}$ & CA vs.UA $=0.025$ & CA vs. $\cup A=0.030^{*}$ \\
\hline HF Norm (nu) & $45.9(17.3)$ & $54.4(16.4)$ & $45.8(17.6)$ & HS vs. $C A=0.031^{*}$ & HS vs. $C A=0.053$ & HS vs. $C A=0.406$ \\
\hline & & & & HS vs. UA $=0.983$ & HS vs. $U A=0.896$ & HS vs. UA $=0.716$ \\
\hline & & & & CA vs.UA $=0.026^{*}$ & CA vs. $U A=0.025^{*}$ & CA vs. UA $=0.030^{*}$ \\
\hline $\mathrm{LnLF} / \mathrm{HF}$ & $0.19(0.76)$ & $-0.19(0.75)$ & $0.22(0.86)$ & HS vs. $C A=0.040^{*}$ & HS vs. $C A=0.054$ & HS vs. $C A=0.438$ \\
\hline & & & & HS vs. $U A=0.838$ & HS vs. UA =0.983 & HS vs. UA $=0.558$ \\
\hline & & & & CA vs. $U A=0.022^{*}$ & CA vs. $U A=0.025^{*}$ & CA vs. $U A=0.029^{*}$ \\
\hline
\end{tabular}

AAT Anti-asthma treatments, CA Controlled asthmatic, HF High frequency, HF Norm normalized high frequency, HRV Heart rate variability, HS Healthy subjects, LF Low frequency, LF Norm normalized low frequency, Ln Natural logarithm, MHR Mean heart rate, SD Standard deviation, TP Total power, UA Uncontrolled asthma, VLF Very low frequency, * Statistically significant.

allergic sensitization to ovalbumin on HRV in a murine model [11]. The researchers were able to demonstrate that sensitized mice had decreased HRV prior to ovalbumin challenge, increased HRV during antigen challenge and finally decreased HRV subsequent to the challenge. Based on these results, HRV is likely to increase in controlled asthmatic patients who usually suffer from acute intermittent exacerbations but not uncontrolled patients, who usually suffer from persistent attacks. This conclusion may explain why TP, LF and HF powers were higher in CA patients examined in this study compared with the UA subjects. Another salient point in this context is that enhanced parasympathetic cardiac autonomic modulations are known to improve, rather than to weaken, HRV $[13,14]$. It appears logical that augmented parasympathetic cardiac autonomic modulations in CA asthmatic patients will improve global HRV, as proved by our results, and not depress HRV, as demonstrated by previous studies.

The difference in the patterns of autonomic modulations between CA and UA patients suggests synonymous variation in the pathophysiological processes involved in each group. In CA patients, enhanced parasympathetic and attenuated sympathetic activity will aggravate bronchoconstriction of the respiratory tree and consequently worsen airways narrowing. According to the present results, parasympathetic autonomic discharge in UA patients is less than CA and comparable to HS suggesting that other mechanisms are involved in inducing airways obstruction in UA patients. Previous reports suggested that UA patients are difficult to treat because they express unique pathological myofilaments in bronchiolar smooth muscles [31, 32] beside other airway changes [33, 34]. In addition, the variations in the pattern of autonomic modulations among asthmatic patients explain differences in the response of asthma to conventional drugs that act on the autonomic nervous system, e.g. UA patients may respond badly to parasympatholytic drugs $[19,20]$.

Although it is widely accepted that the HF power is a reliable indicator of parasympathetic modulations, the validity of the LF power as a measure of sympathetic modulations is a more contentious issue [35]. There is growing evidence that the absolute values of LF are determined by baroreflexes and therefore may efficiently 
reflect parasympathetic tone [36]. The interpretation of the present results was partly based on the normalized LF and HF which, in contrast to their absolute values, are inversely proportional and therefore may better reflect sympathetic and parasympathetic modulations [24]. Another limitation of this study is that respiratory rates were not acknowledged in different study groups while comparing their HRV. HRV is modulated by breathing and evaluation of the rate of respiration in future studies could offer better understanding for autonomic balance in asthmatic patients.

\section{Conclusions}

The present results confirmed that degree of asthma control influences pattern of autonomic modulations/HRV among AS. Enhanced parasympathetic modulations offered higher HRV to CA compared with UA patients who suffered from attenuated vagal tone. However, neither CA nor UA patients were shown to have different autonomic modulations and/or HRV compared with HS. The comparable autonomic modulations/HRV among AS and HS, although different to several previous reports, were based on a well-designed comparison that considered possible influences of MHR and AAT on HRV.

\section{Abbreviations}

AAT: Anti-asthma treatments; ACT: Asthma control test; AS: Asthmatic subjects; BMI: Body mass index; CA: Controlled asthmatic; HF: High frequency; HF Norm: Normalized high frequency; HRV: Heart rate variability; HS: Healthy subjects; LF: Low frequency; LF Norm: Normalized low frequency; Ln: Natural logarithm; MHR: Mean heart rate; Q1: 25 quartile; Q3: 75 quartile; TP: Total power; UA: Uncontrolled asthma; VLF: Very low frequency.

\section{Competing interests}

The author declare that he had no competing interests.

\section{Authors' contributions}

All work was conducted by MFL (study design, data collection, analysis, interpretation and draft writing).

\section{Acknowledgments}

I wish to express my warmest thanks to Dr. A Babikir and Dr. Ramaze F. Elhakeem for their help during the process of data collection.

Received: 19 April 2015 Accepted: 5 October 2015

Published online: 12 October 2015

\section{References}

1. Freeman R, Chapleau MW. Testing the autonomic nervous system. Handb Clin Neurol. 2013;115:115-36.

2. Rubio A, Van Oudenhove L, Pellissier S, Ly HG4, Dupont P, de Micheaux HL, et al. Uncertainty in anticipation of uncomfortable rectal distension is modulated by the autonomic nervous system-a fMRI study in healthy volunteers. Neuroimage. 2015;107:10-22.

3. Harris PR, Sommargren CE, Stein PK, Fung GL, Drew BJ. Heart rate variability measurement and clinical depression in acute coronary syndrome patients: narrative review of recent literature. Neuropsychiatr Dis Treat. 2014;10:1335-47.

4. Jain S, Ton TG, Perera S, Zheng Y, Stein PK, Thacker E, et al. Cardiovascular physiology in premotor Parkinson's disease: a neuroepidemiologic study. Mov Disord. 2012;27(8):988-95.

5. Gomes EL, Sampaio LM, Costa IP, Dias FD, Ferneda VS, Silva GA, et al. Analysis of autonomic modulation during maximal and submaximal work rate and functional capacity in asthmatic children. J Asthma. 2013;50(6):613-8.
6. Gupta J, Dube A, Singh V, Gupta RC. Spectral analysis of heart rate variability in bronchial asthma patients. Indian J Physiol Pharmacol. 2012;56(4):330-6.

7. Tsai YS, Lai FC, Chen SR, Jeng C. The influence of physical activity level on heart rate variability among asthmatic adults. J Clin Nurs. 2011;20(1-2):111-8.

8. Lutfi MF. Autonomic modulations in patients with bronchial asthma based on short-term heart rate variability. Lung India. 2012;29(3):254-8.

9. Garcia-Araújo AS, Pires Di Lorenzo VA, Labadessa IG, Jürgensen SP, Di Thommazo-Luporini L, Garbim CL, et al. Increased sympathetic modulation and decreased response of the heart rate variability in controlled asthma. J Asthma. 2014:31:1-8.

10. Cabiddu R, Aletti F, Duarte Souza V, Peres Costa I, Stirbulov R, Borghi Silva A, et al. Cardiorespiratory coupling during sleep in difficult-to-control asthmatic patients. Conf Proc IEEE Eng Med Biol Soc. 2012;2012:3652-5.

11. Domnik NJ, Seaborn G, Vincent SG, Akl SG, Redfearn DP, Fisher JT. OVAinduced airway hyperresponsiveness alters murine heart rate variability and body temperature. Front Physiol. 2012;3:456. doi:10.3389/fphys.2012.00456. eCollection 2012.

12. Kazuma N, Otsuka K, Matsuoka I, Murata M. Heart rate variability during 24 hours in asthmatic children. Chronobiol Int. 1997;14:597-606.

13. McLachlan CS, Ocsan R, Spence I, Hambly B, Matthews S, Wang L, et al. Increased total heart rate variability and enhanced cardiac vagal autonomic activity in healthy humans with sinus bradycardia. Proc (Bayl Univ Med Cent). 2010;23(4):368-70.

14. Sridhar B, Haleagrahara N, Bhat R, Kulur AB, Avabratha S, Adhikary P. Increase in the heart rate variability with deep breathing in diabetic patients after 12-month exercise training. Tohoku J Exp Med. 2010;220(2):107-13.

15. Task Force of the European Society of Cardiology and the North American Society of Pacing and Electrophysiology. Heart rate variability standards of measurement, physiological interpretation and clinical use. Circulation. 1996:93:65-1043

16. Sacha J. Interaction between heart rate and heart rate variability. Ann Noninvasive Electrocardiol. 2014;19(3):207-16.

17. Eryonucu B, Uzun K, Güler N, Tuncer M, Sezgi C. Comparison of the short-term effects of salmeterol and formoterol on heart rate variability in adult asthmatic patients. Chest. 2005;128(3):1136-9.

18. Anthracopoulos MB, Karatza AA, Davlouros PA, Chiladakis JA, Manolis AS, Beratis NG. Effects of two nebulization regimens on heart rate variability during acute asthma exacerbations in children. J Asthma. 2005;42(4):273-9.

19. Barnes PJ. Severe asthma: advances in current management and future therapy. J Allergy Clin Immunol. 2012;129(1):48-59.

20. Giavina-Bianchi P, Aun MV, Bisaccioni C, Agondi R, Kalil J. Difficult-to-control asthma management through the use of a specific protocol. Clinics. 2010;65(9):905-18.

21. Lutfi MF. Reliability of Asthma Control Test in Assessing Bronchial Asthma; A pilot Study in Sudanese Asthmatic Patients. Alneelain Med J. 2011;1(1):1-7.

22. Kennedy J, Jones S. Asthma Control Test: Reliability, validity, and responsiveness in patients not previously followed by asthma specialists. Pediatrics. 2007;120:S132-3.

23. Miller M, Hankinson J, Brusasco V, Burgos F, Casaburi R, Coates A, et al. Standardisation of spirometry. Eur Respir J. 2005;26:319-38.

24. Lutfi MF. Review article: Heart rate variability. Sud JMS. 2011;6:43-50.

25. Emin O, Esra G, Aysegül D, Ufuk E, Ayhan S, Rusen DM. Autonomic nervous system dysfunction and their relationship with disease severity in children with atopic asthma. Respir Physiol Neurobiol. 2012;183(3):206-10.

26. Sato K, Kawamura T, Abo T. "Senobi" stretch ameliorates asthma symptoms by restoring autonomic nervous system balance. J Investig Med. 2010;58(8):968-70.

27. Ostrowska-Nawarycz L, Wroński W, Błaszczyk J, Buczyłko K, Nawarycz T. The heart rate variability analysis in youth and children with bronchial asthma. Pol Merkur Lekarski. 2006;20(118):399-403.

28. Tascilar E, Yokusoglu M, Dundaroz R, Baysan O, Ozturk S, Yozgat Y, et al. Cardiac autonomic imbalance in children with allergic rhinitis. Tohoku J Exp Med. 2009;219(3):187-91.

29. Boettger MK, Bär KJ, Dohrmann A, Müller H, Mertins L, Brockmeyer NH, et al. Increased vagal modulation in atopic dermatitis. J Dermatol Sci. 2009;53(1):55-9.

30. Du J, He J, Wang Y. A study of heart rate variability in asthma. Zhonghua Jie He He Hu Xi Za Zhi. 2001;24:744-5.

31. Dulin N, Fernandes D, Dowell M, Bellam S, McConville J, Lakser O, et al. What evidence implicates airway smooth muscle in the cause of BHR? Clin Rev Allergy Immunol. 2003;24:73-84.

32. Solway J. What makes the airways contract abnormally? Is it inflammation? Am J Respir Crit Care Med. 2000;161:S164-7. 
33. Brusasco V, Pellegrino R. Invited Review: Complexity of factors modulating airway narrowing in vivo: relevance to assessment of airway hyperresponsiveness. J Appl Physiol. 2003;95:1305-13.

34. Lambert R, Paré P. Lung parenchymal shear modulus, airway wall remodeling, and bronchial hyperresponsiveness. J Appl Physiol. 1997;83:140-7.

35. Reyes del Paso GA, Langewitz W, Mulder L, van Roon A, Duschek S. The utility of low frequency heart rate variability as an index of sympathetic cardiac tone: a review with emphasis on a reanalysis of previous studies. Psychophysiology. 2013;50(5):477-87.

36. Goldstein DS, Bentho O, Park MY, Sharabi Y. Low-frequency power of heart rate variability is not a measure of cardiac sympathetic tone but may be a measure of modulation of cardiac autonomic outflows by baroreflexes Exp Physiol. 2011;96(12):1255-61.

\section{Submit your next manuscript to BioMed Central} and take full advantage of:

- Convenient online submission

- Thorough peer review

- No space constraints or color figure charges

- Immediate publication on acceptance

- Inclusion in PubMed, CAS, Scopus and Google Scholar

- Research which is freely available for redistribution 